Part 3 Galactic foreground science 


\title{
Galactic foreground science: Faraday Tomography at low frequencies
}

\author{
Marijke Haverkorn \\ Department of Astrophysics/IMAPP, Radboud University, Heyendaalseweg 135, 6525 AJ \\ Nijmegen, The Netherlands \\ email: m.haverkorn@astro.ru.nl
}

\begin{abstract}
This contribution describes how low-frequency radio-spectropolarimetric imaging as done for Epoch of Reionization detection is used to investigate the nearby Galactic interstellar medium. The method of Faraday Tomography allows disentangling of every line of sight into various components in Faraday depth, which is a proxy for density-weighted magnetic field. I discuss instrumental biases and side effects of this method, and early results it has yielded.
\end{abstract}

Keywords. ISM: magnetic fields, ISM: structure, techniques: polarimetric, radio continuum: ISM

\section{Introduction}

One of the main challenges of detecting a signal from the Epoch of Reionization is how to either avoid or subtract the foregrounds, as is clear from these Proceedings. One particularly bright foreground is synchrotron emission from the Milky Way Galaxy. However, one person's trash is an other person's treasure, and the Galactic foregrounds that the EoR community is keen to dispose of, are a very valuable source of information for researchers interested in the Galactic magnetized interstellar medium (ISM), in particular in the local interstellar environment of the Sun.

In this contribution, I will describe research on the local ISM using low-frequency radiopolarimetric observations that are also used for EoR detection. Although I focus on lowfrequency spectro-polarimetry to study Galactic foregrounds, this work is supplemented by observations in other tracers and wavelength regimes, which are described elsewhere in this Volume. Indispensable for a complete study of the local magnetized ISM are e.g. observations of submm dust polarization (e..g., Miville-Deschênes, this Volume, Zaroubi et al. (2015), Planck Collaboration et al. (2016), Alves et al. (2018)), optical/near-infrared polarization of starlight (e.g. Pavel (2014)), local dust density (Lallement et al. (2014)) , or neutral hydrogen (Clark et al. (2014), Clark et al. (2015), Clark, this Volume). Also, I will not discuss power spectra of low-frequency polarized radio emission here (e.g. Haverkorn et al. (2003c) Bernardi et al. (2009), Choudhuri, this Volume), but will focus on the technique of Faraday Tomography.

This paper is built up as follows. After a brief partial history of discovery and research of polarized Galactic foregrounds in Section 2, I will introduce the data processing method applied to the low-frequency spectro-polarimetry (EoR) measurements in Section 3 and summarize the current observations in Section 4. I will lay out some of the biggest challenges with this method in Section 5 and discuss how these challenges could be, and are, approached in Section 6. 


\section{A brief history of polarized foreground research}

After the first detection of diffuse polarized radio emission from the Milky Way at $408 \mathrm{MHz}$, now more than half a century ago (Wielebinski \& Shakeshaft (1962), Westerhout et al. (1962)), early Faraday rotation measurements started being used to study the magnetic field structure in the Milky Way (e.g. Muller (1963), Brouw \& Spoelstra (1976), Spoelstra (1984)). For a comprehensive overview of radio-polarimetric and Faraday rotation studies of the diffuse Galactic synchrotron emission, see e.g. Wielebinski (2012).

The richness of polarized Galactic foreground structures without counterparts in total intensity was serendipitously discovered in the late eighties of the last century by Mark Wieringa, then a PhD student at Leiden Observatory supervised by Peter Katgert and Ger de Bruyn. Wieringa's thesis project studied high-redshift radio sources at $327 \mathrm{MHz}$ with the Westerbork Synthesis Radio Telescope (WSRT). When they decided to have a quick look at the polarization properties of the sources in their field, they unexpectedly found degree-long polarized structures that were invisible in their total intensity maps. Wieringa et al. (1993) proposed that these structures originated in Galactic (foreground) synchrotron emission and were caused by small-scale Faraday rotation. As Faraday rotation only causes polarization angle changes but not (necessarily) variations in total intensity, the smooth total intensity signal was filtered out by the interferometer but the small-scale polarization signal was obvious in their maps.

This discovery led to many extensive surveys of polarized radio emission from the Galaxy, such as the all-sky survey of the complete polarized sky at $1.4 \mathrm{GHz}$ at relatively high resolution of 36 arcmin (Wolleben et al. (2006), Testori et al. (2008)), or the polarimetric surveys of the complete Galactic plane at $1.4 \mathrm{GHz}$ at arcminute resolution (Canadian Galactic Plane Survey, Landecker et al. (2010)) and Southern Galactic Plane Survey (Haverkorn et al. (2006)). The WSRT studies at low frequencies $(\sim 350 \mathrm{MHz})$ were continued by a later PhD student of Katgert and De Bruyn (Haverkorn et al. 2003a, 2003b).

A next major step was the description and first application of the method of Rotation Measure Synthesis by Brentjens \& de Bruyn (2005), based on the theory described in Burn (1966). This is the observational method described in this paper, and is introduced in the next section. The study of the magneto-ionized medium in three dimensions by way of RM Synthesis is commonly referred to as Faraday Tomography.

This and other technological and scientific developments led to an increased interest in probing cosmic magnetism with (low-frequency) radio-polarimetry, as is evidenced by the host of broadband spectro-polarimetric surveys underway or planned with modern radio interferometers and single dishes, such as POSSUM (with ASKAP, Gaensler et al. (2010)), GALFACTS (with Arecibo Taylor \& Salter (2010), LOTSS (with LOFAR, Van Eck et al. (2018a)), and VLASS (with VLA, Mao et al. (2014)).

I will concentrate on low frequencies (a few $100 \mathrm{MHz}$ and below), as these are the frequencies relevant for EoR foregrounds. For higher frequency RM Synthesis of Galactic synchrotron emission, see e.g. Wolleben et al. (2010), Sun et al. (2015), and Hill et al. (2017).

\section{Faraday Tomography}

In a magnetized plasma containing both thermal and cosmic ray electrons, synchrotron emission will be emitted and then Faraday rotated throughout the entire volume. This will render the classical observational definition of rotation measure (RM) meaningless, 
which is

$$
\theta=\theta_{0}+\mathrm{RM} \lambda^{2}
$$

where $\theta$ is the observed linear polarization angle, and $\theta_{0}$ is the intrinsic polarization angle at the emission location.

Instead, the polarized synchrotron emission will contain signatures of different Faraday rotation components along the line of sight, which are captured in a Faraday depth spectrum $F(\phi)$. The Faraday depth $\phi$ depends on thermal electron density $n_{e}$ and magnetic field $\mathbf{B}$ and is defined as (Burn (1966)):

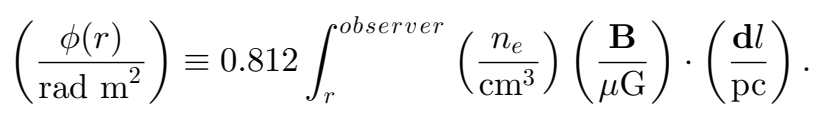

For an emission source separated from the Faraday rotating medium (e.g. pulsar emission being Faraday rotated by the ISM), the values of RM and $\phi$ are equal.

As every Faraday depth component contributes rotation of the polarization angle as a function of $\lambda^{2}$, a Fourier transform of the frequency spectrum of polarized emission with respect to $\lambda^{2}$ will reveal the relative contributions of each Faraday depth component as the Faraday spectrum $F(\phi)$. The Faraday spectrum is thus a Fourier Transform of the complex polarization vector $P\left(\lambda^{2}\right)$. However, as the observed complex polarization vector $\tilde{P}\left(\lambda^{2}\right)$ has a finite $\lambda^{2}$ range, a window function $W\left(\lambda^{2}\right)$ is introduced as $\tilde{P}\left(\lambda^{2}\right)=$ $W\left(\lambda^{2}\right) P\left(\lambda^{2}\right)$. The measured Faraday spectrum $\tilde{F}(\phi)$ can then be computed from the observed polarized radiation as

$$
\begin{aligned}
\tilde{F}(\phi)=F(\phi) * R(\phi) & =K \int_{-\infty}^{+\infty} \tilde{P}\left(\lambda^{2}\right) \mathrm{e}^{-2 i \phi \lambda^{2}} d \lambda^{2} \\
R(\phi) & =K \int_{-\infty}^{+\infty} W\left(\lambda^{2}\right) \mathrm{e}^{-2 i \phi \lambda^{2}} d \lambda^{2} \\
K & =\left(\int_{-\infty}^{+\infty} W\left(\lambda^{2}\right) d \lambda^{2}\right)^{-1}
\end{aligned}
$$

where $R(\phi)$ is commonly called the Rotation Measure Spread Function (RMSF).

Brentjens \& de Bruyn (2005) described this formalism for finite frequency sampling and various spectral dependencies such as power law spectra; these authors then applied the theory to real data the first time. Schnitzeler \& Lee (2015) extended the theory to include arbitrary frequency channel response functions. Since recovery of a Faraday spectrum is necessarily done from a restricted wavelength range, this poses a complex deconvolution problem. Solutions have been proposed in the form of RMCLEAN (Heald (2009)) similar to the Högbom CLEAN algorithm (Högbom (1974)), wavelet-based deconvolution assuming symmetries in the medium (Beck et al. (2012)) or compressive sampling (Li et al. (2011), Andrecut et al. (2012)).

\section{What does Faraday Tomography tell us?}

The 3-dimensional data products resulting from the Faraday Tomography analysis are maps of polarized emission on the sky as a function of Faraday depth (see Equation (3.3)). In principle, this gives us a map of the electron-density-weighted magnetic field component parallel to the line of sight. However, there are a number of effects obfuscating the direct physical interpretation of Faraday Tomography data, in particular artifacts due to the finite frequency coverage of the observations. Since the Fourier Transform to calculate the Faraday spectrum is by definition over a finite $\lambda^{2}$ range, this introduces artifacts and 
uncertainties in the Faraday cube. In addition, broad Faraday depth components in the Faraday spectra are missed, analogous to missing short spacings in an interferometer. At these low frequencies, the Earth's ionosphere also causes a significant Faraday rotation (see e.g. Loi et al. (2015), Mevius et al. (2016)), which is frequency, time, and location dependent and therefore has to be taken into account carefully.

Taking these complicating factors into account, Faraday tomography can give a first idea of scales of magneto-ionic structures in the local ISM, spatial ditributions, and associations with other tracers. Early observational studies of Faraday Tomography focused mainly on description and qualitative interpretation of various Faraday depth components, whereas recent studies are now exploring interpretation, distance allocation, correlations with other components, and/or selection effects.

\subsection{Low-frequency Faraday Tomography data}

In the past decade, a number of studies of low-frequency Faraday Tomography have been published. These studies have explored properties of the polarized emission in these cubes such as morphologies, depolarization characteristics and power spectra.

The demonstration that RM Synthesis worked, with the WSRT around $350 \mathrm{MHz}$, showed extended polarized emission throughout a field centered at the Perseus Cluster, with a large range of Faraday depths from about 0 to $+90 \mathrm{rad} \mathrm{m}^{-2}$ (de Bruyn \& Brentjens (2005)). A wider field around that cluster showed an even broader range of Faraday depths $\left(-50\right.$ to $\left.+100 \mathrm{rad} \mathrm{m}^{-2}\right)$ and proved that this was all due to the Galactic foreground (Brentjens (2011)).

Bernardi et al. (2009) detected for the first time fluctuations in the total and polarized synchrotron emission at frequencies and angular scales relevant for EoR experiments, followed up by Iacobelli et al. (2013) who devised a simple model based on Faraday depth components. Similar models of various superposed synchrotron and Faraday depth components were composed by Pizzo (2010) and Brentjens (2011) for foregrounds towards galaxy clusters, while Jelić et al. (2014), Jelić et al. (2015) studied Galactic foregrounds in actual EoR fields.

What these observations have in common is a bewildering variety of polarization structures, with sizes from the resolution to across the field of view, diverse morphologies over various Faraday depth ranges. Some of these studies show a pervading, (presumed) nearby, low-Faraday-depth component that could be the Local Bubble Wall. Some of these polarization structures are surprisingly straight, often connected to the local magnetic field direction (see Section 6.2).

\section{Challenges in interpretation}

The physical interpretation of Faraday Tomography is a huge challenge. However, with a better understanding of the instrumental biases produced by the method, we can hope to account for these increasingly accurately in order to get through to the information about the actual medium that lies buried in these cubes.

Two of the main challenges are the lack of direct distance information and the insensitivity to Faraday-thick structures, as detailed in the next subsections.

An alternative to RM Synthesis is the method of $Q U$-fitting, avoiding these challenges but introducing others. QU-fitting assumes a certain model for emitting and Faraday rotating components along the line of sight, calculates the expected behavior of Stokes parameters $Q$ and $U$ with wavelength, and fits this to the data to obtain optimum model parameters. This method has been applied to (two-component) radio galaxies (Farnsworth et al. (2011)) and the turbulent intergalactic medium (Ideguchi et al. (2014)). Farnsworth 
et al. (2011) showed that QU-fitting can retrieve Faraday depth components that are undetectable with RM Synthesis. However, applying QU-fitting to the diffuse Galactic foreground would be close to impossible due to the complex, multi-component nature of the medium. As the reliability of QU-fitting depends crucially on the amount of confidence in a certain input model, complex media are as of yet unattainable with this method.

\subsection{Faraday depth and physical distance}

As Faraday depth depends on the direction of the magnetic field component parallel to the line of sight, $B_{\|}$, (Equation (3.2)), it varies very non-linearly with distance. Faraday depth would only increase or decrease monotonically with distance if there are no reversals in $B_{\|}$, which is very unlikely in the turbulent interstellar medium. Therefore, it is not possible to assign a distance to Faraday depth measurements (as is done e.g. for dispersion measures), and a priori it is impossible to say which observed Faraday depth components are closer than others.

\subsection{Insensitivity to Faraday-thick signals}

This instrumental bias intrinsic to the method of RM Synthesis is directly analogous to the insensitivity to large scale structure in an interferometric image due to lack of short spacings down to zero. In RM Synthesis, the lack of short wavelengths down to zero creates an insensitivity for broad structures in Faraday depth space, called Faradaythick structures. The scale in Faraday depth at which sensitivity has dropped by half is

$$
\sim \pi / \lambda_{\min }^{2}
$$

(Brentjens \& de Bruyn (2005)), where $\lambda_{\min }$ is the minimum observing wavelength. Comparing this to the width (FWHM) of the main peak of the RMSF,

$$
\delta \phi \approx 2 \sqrt{3} / \Delta \lambda^{2}
$$

where $\Delta \lambda^{2}$ is the observed bandwidth in wavelength squared, leads to the unfortunate conclusion that for current typical $\Delta \lambda^{2}$ and $\lambda_{\text {min }}$ values, the Faraday depth resolution $\delta \phi$ is comparable to the maximum $\phi$-scale observable. This holds for observations in the meter-length wavelength range, but also typically at L-band in the decimeter-length wavelength range. Thus, effectively, we only detect Faraday-thin structures, i.e. structures narrower than or equally narrow to $\delta \phi$.

\section{Addressing the challenges in interpretation}

\subsection{Understanding biases through simulations}

Van Eck et al. (2017) did basic simulations of the amount of depolarization expected from a Faraday-thick medium. As shown in Brentjens \& de Bruyn (2005), a top-hat function in a Faraday spectrum will only be detectable through a polarized intensity signal at its edges, e.g. for current LOFAR-High Band coverage, only about $\sim 10 \%$ of the original intensity remains (Van Eck et al. (2017)). However, more realistic Faraday-thick distributions (such as an ionized turbulent cloud) will resemble a gaussian function in Faraday space. Van Eck et al. (2017) showed that a Faraday-thick gaussian will be completely depolarized. For typical ISM parameters and decameter observations, a turbulent ionized cloud will not be represented in the Faraday spectrum at all. Instead, these authors suggest that the various Faraday depth components in their data originate from nearby neutral clouds in the local interstellar medium. Since in these neutral clouds, synchrotron 
emissivity can be built up with negligible Faraday rotating, these clouds can be a significant Faraday-thin synchrotron source, i.e. with negligible internal Faraday rotation, rendering them Faraday thin. This hypothesis was recently confirmed in an other field by Jelić et al. (in prep).

\subsection{Assigning distance and finding correlations with other tracers}

Section 4.1 explained that in all Faraday cubes observed, multiple components of polarized emission layers at various Faraday depths occur. By looking for (lack of) similarities in morphology and structure in those multiple components, one can try to assign an order to these. Also, decreasing angular scales of fluctuations in polarized emission at different Faraday depths can be an argument for increasing distance (Pizzo (2010)).

A second method with which distances might be assigned to Faraday depth components is finding associations with various other tracers. Besides allowing distance estimates, studying these associations is also important for understanding the interaction and relative distributions of the various interstellar medium components.

Van Eck et al. (2017) associated two Faraday depth components to two neutral clouds at distances of $\sim 200$ and $\sim 500-800$ pc (using the maps of neutral clouds by Lallement et al. (2014)). Zaroubi et al. (2015) found a surprising correlation between the orientation of Faraday depth filaments and magnetic field directions from polarized Planck emission in one of their three examined fields. Correlations between Faraday depth and dust polarization morphologies have now been confirmed in various fields (see Miville-Deschênes, this Volume, Planck Collaboration et al.(2018)). Although both observables trace magnetic field directions, the correlation between the two is not necessarily expected, since Faraday depth probes the (local) cosmic ray distribution, and dust emission probes the dusty medium along a usually longer line of sight. These correlations may have interesting implications on the amount of mixing of the various interstellar medium components.

A last intriguing connection is that of neutral hydrogen filaments with magnetic field orientation as probed by polarized synchrotron filaments (Kalberla \& Kerp (2016)) or dust polarization (Clark et al. (2014)). Spatial correlation of multiple polarized intensity filaments with HI filaments at different velocities/distances, with multiple orientations in the same field (Kalberla et al. (2017)) opens up a new avenue to assigning distances to polarized filaments by association with HI.

\subsection{Get the global picture: larger sky coverage}

In most high-resolution observed Faraday cubes, Faraday depth structures extend to beyond the primary beam, i.e. exist on degree-scales or larger. MWA observations of larger parts of the sky, at lower resolutions, indicate that these structures can extend to many degrees on the sky (Bernardi et al. (2013), Lenc et al. (2016)).

The logical next step would be to extend spatial coverage at arcminute resolution to map out these structures. Indeed, polarized structures of up to ten degrees in size have been detected (Van Eck et al. (2018b)) in a field of 570 square degrees in the highlatitude sky (Van Eck et al. (2018a), see Figure 1), extending from broad gradients in patches to a very narrow and straight filament exhibiting a Faraday depth gradient perpendicular to its direction. This filament seems to be associated with a long and straight neutral hydrogen filament (Van Eck et al. (2018b)), suggesting an ionized envelope surrounding the neutral filament.

\subsection{Broader frequency coverage}

Lastly, as the sensitivity to Faraday thickness and the Faraday depth resolution depend on the wavelength coverage according to Equations (5.1) and (5.2), extending the bandwidth 


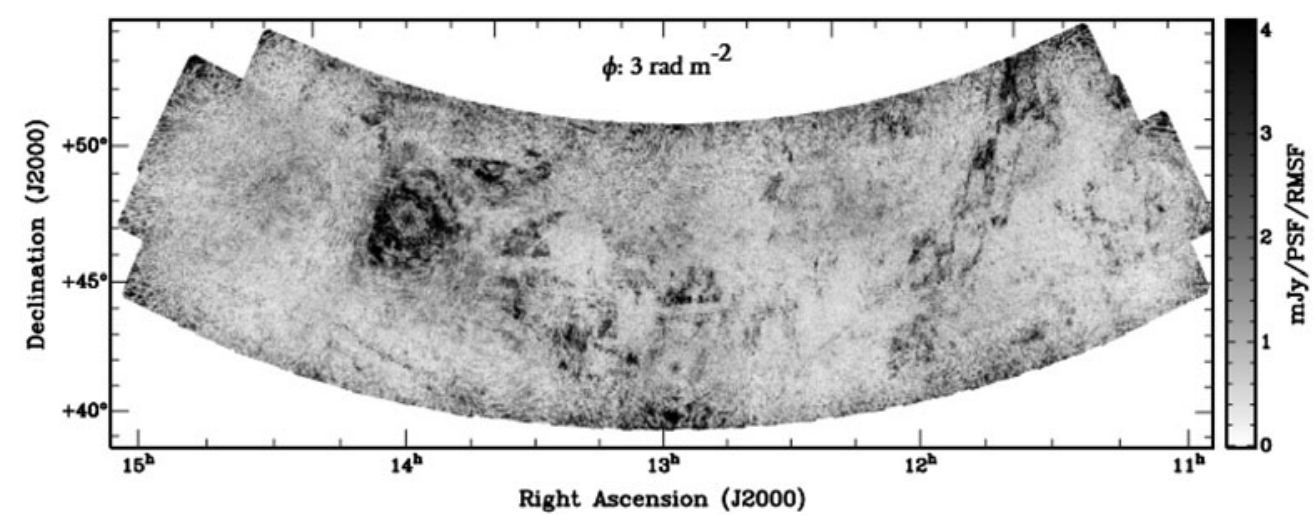

Figure 1. This Faraday depth slice at $\phi=3 \mathrm{rad} \mathrm{m}^{-2}$ (Van Eck et al. (2018b)) shows a wide variety of mostly filamentary polarized structure across the field. Note that the bright circular structure centered at $\mathrm{RA} \sim 14 \mathrm{~h}$, dec $\sim 47.5^{\circ}$ is instrumental polarization caused by a bright extragalactic source.

to smaller wavelength will increase sensitivity to large scales while keeping a high Faraday depth resolution.

Also, as Faraday-thickness depends on wavelength, analysis of Faraday depth structures at various wavelength provides a more complete image, as shown in the comparison of $150 \mathrm{MHz}$ and $350 \mathrm{MHz}$ data by Jelić et al. $(2014,2015)$.

A large project which is expanding the bandwidth significantly throughout the lower radio frequencies is the Global Magneto-Ionic Medium Survey (GMIMS, Wolleben et al. (2009), PI Tom Landecker). GMIMS consists of 6 separate surveys: one survey spanning approximately the frequency intervals of $300-800 \mathrm{MHz}, 800-1300 \mathrm{MHz}$ and $1300-$ $1800 \mathrm{MHz}$ each, for each hemisphere. At this moment, three of these six surveys are in (fairly to very) advanced stages:

- SOUTH-LOW: the Southern hemisphere survey from $300-800 \mathrm{MHz}$ has been observed with the Parkes $64 \mathrm{~m}$ single-dish telescope, but is heavily affected by RFI.

- SOUTH-HIGH: the Southern hemisphere survey from $1300-1800 \mathrm{MHz}$ was also observed with the Parkes telescope. Since this survey was independently initiated, it is also referred to as the Southern Twenty-cm All-sky Polarization Survey (STAPS).

- NORTH-HIGH: the Northern hemisphere survey from $1270-1750 \mathrm{MHz}$ data was observed with the DRAO 26-m telescope. Part of this survey has already resulted in the first scientific results from GMIMS (Wolleben et al. (2010), Sun et al. (2015) and Hill et al. (2017)).

\section{Conclusions}

Faraday Tomography is a very promising analysis technique to investigate the local magnetized interstellar medium. To fully exploit this method, more understanding of the unwanted effects of the method on the data is necessary. Already, Faraday Tomography of the low-frequency Galactic synchrotron radiation has revlead intriguing magnetized structures of various morphologies, some of which show clear correlations with neutral hydrogen filaments or magnetic field orientations from dust polarization. 


\section{Acknowledgments}

I would like to acknowledge Cameron Van Eck and Vibor Jelić for valuable comments on earlier versions of this manuscript.

In addition, I want to express my gratitude to Ger de Bruyn for half a lifetime of collaboration and friendship. Ger, who unfortunately passed away three months before this conference, was one of the drivers of the LOFAR EoR effort, but also (decades earlier) one of the pioneers of diffuse Galactic polarization research. From the start of my $\mathrm{PhD}$ project until now, Ger has always be a source of motivation and energy, a role model for attention to depth and details, and an inexhaustible originator of ideas. Still, my work gets better whenever I seriously think about the question: "What would Ger do?".

\section{References}

Alves, M. I. R., Boulanger, F., Ferrière, K., Montier, L., submitted to $A \mathscr{E} A$

Andrecut, M., Stil, J. M., \& Taylor, A. R. 2012, AJ, 143, 33

Beck, R., Frick, P., Stepanov, R., \& Sokoloff, D. 2012, A\& $A$, 543, A113

Bernardi, G., de Bruyn, A. G., Brentjens, M. A., et al. 2009, A\&A 500, 965

Bernardi, G., Greenhill, L. J., Mitchell, D. A., et al. 2013, ApJ, 771, 105

Bingham, R. G. \& Shakeshaft, J. R. 1967, MNRAS, 136, 347

Brentjens, M. A., \& de Bruyn, A. G. 2005, A\& $A, 441,1217$

Brentjens, M. A. 2011, A\&A, 526, 9

Brouw, W. N. \& Spoelstra, T. A. T. 1976, A\&AS, 26, 129

Burn, B. J. 1966, MNRAS, 133, 67

de Bruyn, A. G. \& Brentjens, M. A. 2005, A\&A, 441, 931

Clark, S. E., Hill, J. C., Peek, J. E. G., Putman, M. E., \& Babler, B. L. 2015, PRL, 115, 241302

Clark, S. E., Peek, J. E. G., \& Putman, M. E. 2014, ApJ 789, 82

Farnsworth, D., Rudnick, L., \& Brown, S. 2011, AJ, 141, 191

Gaensler, B. M., Landecker, T. L., \& Taylor, A. R., POSSUM Collaboration 2010, Bulletin of the American Astronomical Society, 42, 470.13

Haverkorn, M., Gaensler, B. M., McClure-Griffiths, N. M., Dickey, J. M., \& Green, A. J. 2006, ApJS, 167, 230

Haverkorn, M., Katgert, P., \& de Bruyn, A. G. 2003, A\&A, 403, 1031

Haverkorn, M., Katgert, P., \& de Bruyn, A. G. 2003, A\&A, 404, 233

Haverkorn, M., Katgert, P., \& de Bruyn, A. G. 2003, A\& A, 403, 1045

Heald, G. 2009, Cosmic Magnetic Fields: From Planets, to Stars and Galaxies, 259, 591

Hill, A. S., Landecker, T. L., Carretti, E., et al. 2017, MNRAS, 467, 4631

Högbom, J. A. 1974, A\&AS, 15, 417

Iacobelli, M., Haverkorn, M., \& Katgert, P. 2013, A\&A, 549, A56

Ideguchi, S., Takahashi, K., Akahori, T., Kumazaki, K., \& Ryu, D. 2014, PASJ, 66, 5

Jelić, V., de Bruyn, A. G., Mevius, M., et al. 2014, A\&A, 568, A101

Jelić, V., de Bruyn, A. G., Pandey, V. N., et al. 2015, A\&\&A, 583, A137

Kalberla, P. M. W., Kerp, J., Haud, U., \& Haverkorn, M. 2017, A\&SA, 607, A15

Kalberla, P. M. W. \& Kerp, J. 2016, A\&A, 595, A37

Lallement, R., Vergely, J.-L., Valette, B., et al. 2014, A\&A, 561, A91

Landecker, T. L., Reich, W., Reid, R. I., et al. 2010, $A \& A$, 520, A80

Lenc, E., Gaensler, B. M., Sun, X. H., et al. 2016, ApJ, 830, 38

Li, F., Brown, S., Cornwell, T. J., \& de Hoog, F. 2011, A\&A, 531, A126

Loi, S. T., Murphy, T., Bell, M. E., et al. 2015, MNRAS, 453, 2731

Mao, S. A., Banfield, J., Gaensler, B., et al. 2014, arXiv:1401.1875

Mevius, M., van der Tol, S., Pandey, V. N., et al. 2016, Radio Science, 51, 927

Muller, C. A. 1963, Nature, 200, 155

Pavel, M. D. 2014, AJ, 148, 49

Pizzo, R. F. 2010, Ph.D. Thesis, Groningen University 
Planck Collaboration, Akrami, Y., Ashdown, M., et al. 2018, arXiv:1801.04945

Planck Collaboration, Adam, R., Ade, P. A. R., et al. 2016, A\&A, 586, A135

Schnitzeler, D. H. F. M. \& Lee, K. J. 2015, MNRAS, 447, L26

Spoelstra, T. A. T., 1984 A\& A, 135, 238

Sun, X. H., Landecker, T. L., Gaensler, B. M., et al. 2015, ApJ, 811, 40

Taylor, A. R. \& Salter, C. J. 2010, The Dynamic Interstellar Medium: A Celebration of the Canadian Galactic Plane Survey, 438, 402

Testori, J. C., Reich, P., \& Reich, W. 2008, AछA, 484, 733

Uyaniker, B., Fuerst, E., Reich, W., Reich, P., \& Wielebinski, R. 1998, A\&SAS, 132, 401

Van Eck, C. L., Haverkorn, M., Alves, M. I. R., et al. 2018, arXiv:1801.04467

Van Eck, C. L., Haverkorn, M., Alves, M. I. R., et al. 2017, A\&A, 597, A98

Van Eck, C. L., Haverkorn, M., Alves, M. I. R., et al. 2018, submitted to $A \mathscr{G} A$

Westerhout, G., Seeger, Ch. L., Brouw, W. N., \& Tinbergen, J. 1962, BAN, 16, 187

Wielebinski, R. 2012, Journal of Astronomical History and Heritage, 15, 76

Wielebinski, R. \& Shakeshaft, J. R. 1962, Nature, 195, 982

Wieringa, M. H., de Bruyn, A. G., Jansen, D., Brouw, W. N., \& Katgert, P. 1993, A\&A, 268, 215

Wolleben, M., Fletcher, A., Landecker, T. L., et al. 2010, ApJL, 724, L48

Wolleben, M., Landecker, T. L., Carretti, E., et al. 2009, Cosmic Magnetic Fields: From Planets, to Stars and Galaxies, 259, 89

Wolleben, M., Landecker, T. L., Reich, W., \& Wielebinski, R. 2006, A\&\&A, 448, 411

Zaroubi, S., Jelić, V., de Bruyn, A. G., et al. 2015, MNRAS 454, L46 